\title{
The impact of periampullary diverticula on the endoscopic treatment of choledocholithiasis
}

\author{
Fatih Aslan, Mahmut Arabul, Emrah Alper, Mustafa Celik, Altay Kandemir, Belkis Unsal \\ Department of Gastroenterology, Atatürk Research and Training Hospital, izmir, Turkey
}

Prz Gastroenterol 2012; 7 (5): 281-285

DOI: $10.5114 /$ pg.2012.32066

Key words: periampullary diverticula, endoscopic retrograde cholangiopancreatography, choledocholithiasis.

\begin{abstract}
Address for correspondence: Mahmut Arabul, Department of Gastroenterology, Izmir Ataturk Training and Research Hospital, İzmir Atatürk Eğitim ve Araștırma Hastanesi, 35160, Karabağlar, İzmir, phone: 0090.232.244 44 44, mobile phone: 0090.505 .4976032 , e-mail: mahmutarabul@gmail.com
\end{abstract}

\begin{abstract}
Introduction: Periampullary diverticula (PD) are generally asymptomatic and frequently diagnosed coincidentally during endoscopic retrograde cholangiopancreatography (ERCP).

Aim: To investigate the impact of PD on procedural success in ERCP for common bile duct (CBD) stones, and possible complications.

Material and methods: Five hundred and eighty-six patients diagnosed with CBD stones by diagnostic techniques and treated by ERCP were prospectively included in the study. Periampullary diverticula presence (patients with PD were allocated to group $A$, and those without PD to group B), size of the diverticulum $(<2 \mathrm{~cm}$ or $\geq 2 \mathrm{~cm})$, papillary association with the diverticulum, size and number of CBD stones, were prospectively recorded for each patient. Total extraction of CBD stones by ERCP was considered successful treatment, and inability to remove the stone(s) was defined as lack of success.

Results: Group A consisted of 478 (82\%), and group B 108 patients (18\%). Cannulation success at first try was $97.7 \%$ in group A, and $95.4 \%$ in group B patients $(p>0.05)$. The prevalence of stone impaction was $3.1 \%$ in group $A$, and $10.2 \%$ in group B $(p<0.01) .86 .8 \%$ of group A and $81.5 \%$ of group B patients were treated endoscopically.

Conclusions: Periampullary diverticula is a contributing factor in the formation of choledocholithiasis, and in the increase in size or impaction of existing stones. However, the presence of PD does not necessarily affect the success of ERCP or the prevalence of procedure-related complications in patients with CBD stones.
\end{abstract}

\section{Introduction}

Periampullary diverticula (PD) are generally located along the medial aspect of the second portion of the duodenum. Incidence increases with age. Radiological and postmortem studies report a prevalence rate of $5 \%$, while endoscopic prevalence is $5-32 \%[1,2]$. Periampullary diverticula are usually asymptomatic, but there exist studies that report an increased prevalence with choledocholithiasis and pancreatic disorders [3-5].

Several studies have demonstrated that periampullary diverticula in patients undergoing endoscopic retrograde cholangiopancreatography (ERCP) procedures have an impact on procedural success, success of cannulation, and inter- and post-procedure complications. However, these results are considered to be controversial due to the small number of patients and the differences in study designs $[6,7]$.
Aim

In this study, we aimed to investigate the impact of PD on procedural success of ERCP for common bile duct (CBD) stones, and procedure-related complications.

\section{Material and methods}

Five hundred and eighty-six patients who presented to the Gastroenterology Clinic of Izmir Atatürk Training and Research Hospital between September 2008 and September 2010 with clinical and laboratory findings of obstructive jaundice and who underwent ERCP with a diagnosis of CBD stone by abdominal ultrasonography, magnetic resonance cholangiopancreatography (MRCP) or endoscopic ultrasonography (EUS) were prospectively included in the study. Patients with acute cholangitis and acute pancreatitis, pregnancy, or history of gastric procedures were excluded. Patients' demographic char- 
acteristics and biochemical findings (alanine aminotransferase, aspartate aminotransferase, alkaline phosphatase, serum total bilirubin and amylase levels) were recorded.

Endoscopic retrograde cholangiopancreatography procedures were performed by two physicians with 600 procedures/year/9 years experience using an Olympus TJF 145 standard therapeutic duodenoscope and a Shimadzu Opescope 50N fluoroscopy scope. Written and verbal informed consent was obtained from patients prior to the procedure. Endoscopic retrograde cholangiopancreatography was performed following 12-hour fasting under respiratory and cardiac monitoring, with 3-4\% nasal $\mathrm{O}_{2}$ administration, under sedative anesthesia (propofol 1-2 mg/kg, midazolam 0.01-0.1 mg/kg). After visualization of the papilla by the duodenoscope, cannulation was achieved with a standard sphincterotome. In cases of failure of biliary cannulation after five tries during the same session, the precut technique or cannulation by fistulotomy was attempted. The procedure was terminated upon failure of cannulation by the precut technique. A second ERCP procedure was performed on these patients within $48 \mathrm{~h}$. Unsuccessful cannulation during the second procedure was considered treatment failure. Following CBD cannulation, fluoroscopic imaging was obtained with the aid of contrast agents. The location, size, number, and impaction of stones were determined. Following sufficient endoscopic sphincterotomy, stone extraction by means of an ERCP balloon was attempted. In patients where this method failed, a mechanical lithotriptor was used. In cases where balloon sweeping and mechanical lithotriptor failed, a plastic biliary stent (7- to 10-French diameter, $10-12 \mathrm{~cm}$ length, according to the location and size of stones) was placed. Complete removal of CBD stones by ERCP was considered "treatment success", and inability to remove stones was considered "treatment failure".

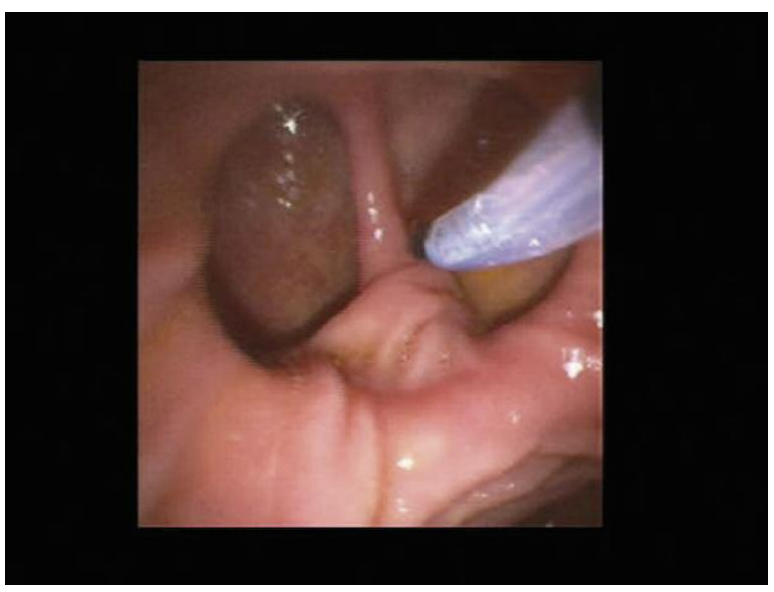

Fig. 1. Small diverticula
A periampullary diverticulum was defined as a $>5 \mathrm{~mm}$ depression of the mucosa within a $2.5-\mathrm{cm}$ radius of the papilla in endoscopic imaging by a duodenoscope [8]. Patients with and without diverticula were allocated to two groups (group B and group A, respectively). Group B patients were further divided into two groups according to diverticulum diameter: those with a diameter of $<2 \mathrm{~cm}$ were recorded as patients with small diverticula (group I), while those with a diameter of $\geq 2 \mathrm{~cm}$ were recorded as patients with large diverticula (group II) (Figures 1 and 2). Patients were also stratified according to the location of the papilla - at the edge of the diverticulum or within the diverticulum.

The duration of the ERCP procedure, cannulation success, inter- and post-procedure complications (bleeding, perforation, post-procedure symptomatic pancreatitis), size and number of CBD stones, stone impaction, treatment technique (balloon only, balloon and basket, plastic stent placement, endoscopic treatment in a separate session, and surgery) were recorded and evaluated.

\section{Statistical analysis}

Statistical analysis were performed using the SPSS17.0 package, using Student's $t$-test, $\chi^{2}$ test, and Fisher's exact test.

\section{Results}

Group A (without diverticula) consisted of 478 patients (82\%), group B (with diverticula) of 108 patients $(18 \%)$. Mean age was significantly higher in patients with diverticula ( $p=0.0001)$. There was no correlation between the groups in terms of gender $(p<0.05)$ (Table I).

Cannulation was successful during the first ERCP session in 467 patients (97.7\%) in group A, and 103 patients $(95.4 \%)$ in group B. There was no difference be-

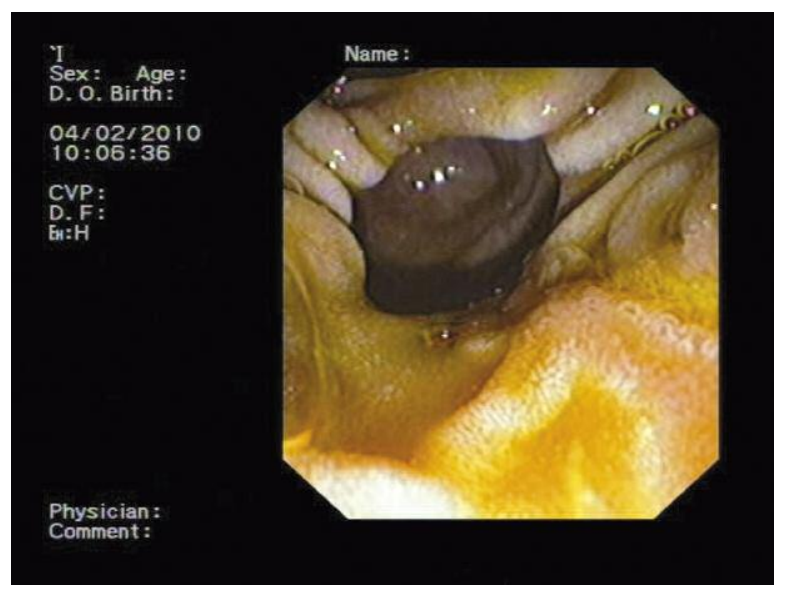

Fig. 2. Large diverticula 
tween the groups in terms of cannulation technique or success $(p=0.52)$. There was also no difference between the groups in terms of the requirement for a second ERCP procedure $(p=0.35)$ (Table I).

The papilla was located at the edge of the diverticulum in 67 patients (62\%), and within the diverticulum in 41 (38\%). The size of the diverticulum was smaller than $2 \mathrm{~cm}$ in 90 patients (83.3\%), and $\geq 2 \mathrm{~cm}$ in 18 (16.7\%). Among the 18 patients with large diverticula, the papilla was located within the diverticulum in 12 , and at the edge of the diverticulum in $6(p=0.007)$ (Table II).

There was no difference between the groups in terms of the number of CBD stones. Average stone diameter was larger in patients with diverticula compared with those without diverticula ( $p=0.001)$, and the prevalence of stone impaction was higher $(10.2 \%$ in group A vs. $3.1 \%$ in group B; $p=0.003$ ) (Table I). Among patients with impacted stones, average size of stone was $15 \pm 6 \mathrm{~mm}$ in group $A$ and $22 \pm 11 \mathrm{~mm}$ in group $B$ patients $(p=0.06)$. There was no correlation between the size of stones and the diameter of diverticula or

Table I. Comparison of patients with and without diverticula

\begin{tabular}{|c|c|c|c|}
\hline Parameter & Group A & Group B & Value of $p$ \\
\hline Patients, $n(\%)$ & $478(82)$ & $108(18)$ & \\
\hline Age, mean \pm SD [years] & $62 \pm 17$ & $74 \pm 11$ & $<0.001$ \\
\hline Gender (M/F) & $193 / 285$ & $41 / 67$ & $>0.05$ \\
\hline Cannulation success, $n$ (\%) & 467 (97.7) & $103(95.4)$ & $>0.05$ \\
\hline \multicolumn{4}{|l|}{ Cannulation type, $n(\%)$ : } \\
\hline Standard cannulation & $471(98.5)$ & $106(98.1)$ & $>0.05$ \\
\hline Precut & $7(1.5)$ & $2(1.7)$ & $>0.05$ \\
\hline $\begin{array}{l}\text { Maximum stone diameter, } \\
\text { mean } \pm S D[\mathrm{~mm}]\end{array}$ & $12 \pm 7$ & $15 \pm 8$ & $<0.01$ \\
\hline \multicolumn{4}{|l|}{ Number of stones, $n(\%)$ : } \\
\hline 1 & $253(52.9)$ & $49(45.4)$ & $>0.05$ \\
\hline 2 & $66(13.8)$ & $17(15.7)$ & $>0.05$ \\
\hline 3 & $34(7.1)$ & $8(7.4)$ & $>0.05$ \\
\hline$>3$ & $125(26.2)$ & $34(31.5)$ & $>0.05$ \\
\hline Stone impaction, $n(\%)$ & $15(3.1)$ & $11(10.2)$ & $<0.01$ \\
\hline Repeat ERCP, $n(\%)$ : & $92(19.2)$ & $30(27.8)$ & $>0.05$ \\
\hline Cannulation failure & $11(12)$ & $5(16.7)$ & $>0.05$ \\
\hline Desaturation & $5(5.4)$ & 0 & $>0.05$ \\
\hline Failure to extract stone & $30(32.6)$ & $14(46.7)$ & $>0.05$ \\
\hline Residual stone & $46(50)$ & $11(36.7)$ & $>0.05$ \\
\hline $\begin{array}{l}\text { Complication (bleeding), } \\
n(\%)\end{array}$ & $20(4.2)$ & $8(7.4)$ & $>0.05$ \\
\hline Treatment success, $n$ (\%) & $415(86.8)$ & $88(81.5)$ & $>0.05$ \\
\hline Treatment failure, $n(\%)$ & $63(13.2)$ & $20(18.5)$ & $>0.05$ \\
\hline
\end{tabular}

location of papilla ( $p=0.174$ and $p=0.7$, respectively) (Tables II and III)

Endoscopic stone removal was successful in 503 (86\%) of the total 586 patients, and unsuccessful in 83 (14\%) $(p=0.134)$. Treatment success was achieved in 415 patients (86.8\%) in group A and 88 (81.5\%) in group B, while attempts at stone extraction failed in 63 patients $(13.2 \%)$ in group A and $20(18.5 \%)$ in group B $(p=0.15)$. There was no correlation between success of endoscopic treatment and size of diverticula or location of papilla (81\% in group I, $83 \%$ in group II; $p=0.26$ ) (Table II). Endoscopic treatment was successful in 10 group A patients $(67 \%)$ and 5 group B patients (45\%) with impacted stones $(p=0.25)$ (Table III).

There was sphincterotome-related bleeding in $28(4.7 \%)$ of the total 586 patients $(20(4.2 \%)$ in group A and $8(7.4 \%)$ in group $B ; p=0.12)$. No complications occurred in patients treated with the precut technique. There was no perforation. No correlation was determined between the risk of bleeding and size of diverticula or location of papilla $(p>0.05)$.

Table II. Comparison of patient results according to size of diverticula

\begin{tabular}{|c|c|c|c|}
\hline Parameter & Group I & Group II & Value of $p$ \\
\hline Patients, $n(\%)$ & $90(83 \%)$ & $18(17 \%)$ & \\
\hline Age, mean \pm SD [years] & $74 \pm 11$ & $78 \pm 7$ & $<0.05$ \\
\hline Gender (M/F) & $36 / 54$ & $5 / 13$ & $>0.05$ \\
\hline \multicolumn{4}{|l|}{ Location of Papilla, $n(\%)$ : } \\
\hline $\begin{array}{l}\text { Papilla at edge } \\
\text { of diverticulum }\end{array}$ & $61(67.8)$ & $6(33.3)$ & $<0.01$ \\
\hline Papilla within diverticulum & $29(32.2)$ & $12(66.7)$ & $<0.01$ \\
\hline $\begin{array}{l}\text { Size of stone, } \\
\text { mean } \pm \text { SD }[\mathrm{mm}]\end{array}$ & $14 \pm 8$ & $16 \pm 9$ & $>0.05$ \\
\hline Stone impaction, $n(\%)$ & $8(8.8)$ & $3(16.6)$ & $>0.05$ \\
\hline \multicolumn{4}{|l|}{ Cannulation type, $n$ (\%): } \\
\hline Standard cannulation & $90(100)$ & $16(88.9)$ & $>0.05$ \\
\hline Precut & 0 & $2(11.1)$ & \\
\hline Treatment success, $n(\%)$ & $73(81)$ & $15(83)$ & $>0.05$ \\
\hline Treatment failure, $n(\%)$ & $17(19)$ & $3(17)$ & $>0.05$ \\
\hline
\end{tabular}

Table III. Results of patients with stone impaction

\begin{tabular}{lccc} 
Parameter & Group A & Group B & Value of $p$ \\
\hline Patients, $n(\%)$ & $15(3.1)$ & $11(10.2)$ & $<0.05$ \\
\hline Age, mean \pm SD [years] & $61 \pm 23$ & $80 \pm 10$ & $<0.01$ \\
\hline Gender (M/F) & $4 / 11$ & $5 / 6$ & $>0.05$ \\
\hline Size of stone, mean \pm SD [mm] & $15 \pm 6$ & $22 \pm 11$ & $>0.05$ \\
\hline Treatment success, $n(\%)$ & $10(67)$ & $5(45)$ & $>0.05$ \\
\hline Treatment failure, $n(\%)$ & $5(33)$ & $6(55)$ & $>0.05$
\end{tabular}




\section{Discussion}

Periampullary diverticula are usually acquired lesions. They are quite uncommon under the age of forty, and their prevalence increases with age. Advanced age contributes to the development of PD due to weakening of regional connective tissue [9]. A prevalence of 5-32\% has been reported in patients undergoing duodenoscopy, and prevalence increases with age and with female gender $[1,4,10]$. In a study on 350 patients, Zoepf et al. reported a PD incidence of $12.5 \%$, and higher average age of patients with PD compared to those without PD [3]. A study by Panteris et al. conducted on 601 patients reported a PD incidence of $20.7 \%$ and a mean age of 72 years [6]. In both studies, although PD was more prevalent in female patients, the difference was non-significant when compared with patients without PD. We also found a PD prevalence of $18.4 \%$ within our patient population, and the mean age of patients with PD was significantly higher than patients without diverticula $(p<0.001)$.

There is conflicting evidence regarding the impact of periampullary diverticula on the success of ERCP cannulation; some authors report an impact [11, 12], others a lack of impact $[2,6,8,13]$. Lobo et al. reported a cannulation success rate of $62.4 \%$ in patients with PD, and $92.7 \%$ in those without PD [11]. In another study, the reported success rate was $95.4 \%$ in patients with PD, and $98.9 \%$ in patients without PD [3]. This difference might be attributed to the definition of treatment success (e.g., if failure to cannulate during the initial ERCP session was considered treatment failure or if alternative cannulation attempts such as the precut technique were not used). We determined that the presence of diverticula or the size of the diverticulum has no effect on cannulation success. In the majority of previously published studies, patient groups are not homogeneous [3, 6, 8]. Any condition invading the papilla (periampullary tumors, pancreatic cancer invasion, etc.) has a negative impact on cannulation success and increases the risk of procedure-related complications. We only enrolled patients in whom CBD stones had been diagnosed by non-invasive (ultrasonography or $M R C P$ ) or minimally invasive (EUS) methods, on whom ERCP had been performed. We believe that the team performing the ERCP procedures being highly experienced, the procedures being performed under sedative anesthesia administered by a specialist, as well as the diversity and adequacy of the equipment at hand (fine-tipped sphincterotome, thin guide wire, needle knife with and without guide, etc.), contributed to the similar and high rate of cannulation in both patient groups. Furthermore, the presence of diverticula might result in the dysfunction of the sphincter of Oddi [14], which would reduce resistance against the sphincterotome, thereby enhancing cannulation success. Periampullary diverticula might affect cannulation success through anatomical damage to the location of the papillary ostium [3, 11, 12]. In the study by Zoepf et al., the location of the papilla in patients with failed cannulation was inside the diverticulum in $62.5 \%$ and outside the diverticulum in $25 \%$ [3]. In another study, the location of the papilla was inside the diverticulum in $62 \%$ and outside the diverticulum in $22 \%$ of patients with failed cannulation [11]. We could not establish any correlation between the location of the papilla and cannulation success. The relatively high rate of cannulation success in our cohort might be attributed to the extradiverticular location of the papilla in the majority of our patients. The location of the papillary ostium may differ according to the size of the diverticulum. This might inhibit deep cannulation by a standard sphincterotome, or restrict safe sphincterotomy after cannulation [7] since a large diverticulum or the papilla being located within a large diverticulum would theoretically make cannulation more difficult due to the luminal defect, and also facilitate sphincterotomy-related complications $[2,3,7]$. In the study by Zoepf et al., papillary location was $54.9 \%$ within the diverticulum and $37.7 \%$ at the edge of the diverticulum, while the incidence of bleeding was $8.8 \%$ in the patient group and $4.8 \%$ in the control group $(p<0.05)$. In our study, there was no difference between the groups in terms of sphincterotomy-related bleeding $(p>0.05)$. This discrepancy might be attributed to the majority (62\%) of our group B patients having an extra-diverticular papillary location. We could not establish a relationship between bleeding and the presence of diverticula, the size of the diverticulum or papillary location. We thus conclude that in patients undergoing ERCP for CBD stones, the presence of diverticula has no impact on procedure success or the development of complications.

Periampullary diverticula, besides creating external compression on the bile duct and thus inhibiting biliary drainage, cause bile stasis through anatomical dysfunction of the papilla or the sphincter of Oddi. This has a major impact on the development of CBD stones [11, 12, 14-19]. However, the size and impaction of stones are not specified in previously published studies. Large or impacted stones may in fact not only have an impact on the success of the procedure, but may also restrict endoscopic success through the prevention of complete stone removal. In the group with diverticula in our study, although both the rate of stone impaction and the average size of the largest stone was higher than the group without diverticula $(p<0.01)$, there was no difference 
between the groups in terms of endoscopic treatment success $(p>0.05)$. Lobo et al. reported successful cannulation in the presence of biliary stones. In this study on 30 patients, 11 (36.6\%) were treated surgically, 12 endoscopically, 6 were administered stents and 1 received no treatment [8]. In another recent study, 28 of 30 patients with choledocholithiasis and diverticula were treated endoscopically [8]. In our study, there was no difference between patient groups in terms of endoscopic treatment success $(p>0.05)$. The lower rate of treatment failure in our study, besides the experienced ERCP team, might be due to developments in equipment used for stone extraction with ERCP and the increased availability of this equipment.

\section{Conclusions}

Periampullary diverticula have a major impact on the formation of choledocholithiasis, and in the increase in size or impaction of existing stones. However, the presence of periampullary diverticula in patients with biliary stones does not affect the success of ERCP, the prevalence of procedure-related complications, or the rate of endoscopic treatment success in the hands of experienced physicians. The negative impact of periampullary diverticula on the therapeutic procedure might be related to the increased incidence, and comorbidities, with age.

\section{References}

1. Kirk AP, Summerfield JA. Incidence and significance of juxtapapillary diverticula at endoscopic retrograde cholangiopancreatography. Digestion 1980; 20: 31-5.

2. Boix J, Lorenzo-Zúñiga $V$, Añaños F, et al. Impact of periampullary duodenal diverticula at endoscopic retrograde cholangiopancreatography: a proposed classification of periampullary duodenal diverticula. Surg Laparosc Endosc Percutan Tech 2006; 16: 208-11.

3. Zoepf T, Zoepf DS, Arnold JC, et al. The relationship between juxtapapillary duodenal diverticula and disorders of the biliopancreatic system: analysis of 350 patients. Gastrointest Endosc 2001; 54: 56-61.

4. Leivonen MK, Halttunen JA, Kivilaakso EO. Duodenal diverticulum at endoscopic retrograde cholangiopancreatography, analysis of 123 patients. Hepatogastroenterology 1996; 43: 961-6.

5. Uomo G, Manes G, Ragozzino A, et al. Periampullary extraluminal duodenal diverticula and acute pancreatitis: an underestimated etiological association. Am J Gastroenterol 1996; 91 1186-8.

6. Panteris V, Vezakis A, Filippou G, et al. Influence of juxtapapillary diverticula on the success or difficulty of cannulation and complication rate. Gastrointest Endosc 2008; 68: 903-10.

7. Boender J, Nix GA, de Ridder MA, et al. Endoscopic papillotomy for common bile duct stones: factors influencing the complication rate. Endoscopy 1994; 26: 209-16.

8. Tyagi P, Sharma P, Sharma BC, Puri AS. Periampullary diverticula and technical success of endoscopic retrograde cholangiopancreatography. Surg Endosc 2009; 23: 1342-5.
9. Løtveit T, Skar V, Osnes M. Juxtapapillary duodenal diverticula. Endoscopy 1988; 20 Suppl 1: 175-8.

10. Kim MH, Myung SJ, Seo DW, et al. Association of periampullary diverticula with primary choledocholithiasis but not with secondary choledocholithiasis. Endoscopy 1998; 30: 601-4.

11. Lobo DN, Balfour TW, Iftikhar SY. Periampullary diverticula: consequences of failed ERCP. Ann R Coll Surg 1998; 80: 326-31.

12. Rajnakova A, Goh PM, Ngoi SS, Lim SG. ERCP in patients with periampullary diverticulum. Hepatogastroenterology 2003; 50: 625-8.

13. Tham TC, Kelly M. Association of periampullary duodenal diverticula with bile duct stones and with technical success of endoscopic retrograde cholangiopancreatography. Endoscopy 2004; 36: 1050-3

14. Viceconte G, Viceconte GW, Bogliolo G. Endoscopic manometry of the sphincter of Oddi in patients with and without juxtapapillary duodenal diverticula. Scand I Gastroenterol 1984; 19: 329-33.

15. Kennedy RH, Thompson MH. Are duodenal diverticula associated with choledocholithiasis? Gut 1988; 29: 1003-6.

16. Hagège $H$, Berson $A$, Pelletier $G$, et al. Association of juxtapapillary diverticula with choledocholithiasis but not with cholecystolithiasis. Endoscopy 1992; 24: 248-51.

17. Wu SD, Su Y, Fan Y, et al. Relationship between intraduodenal peri-ampullary diverticulum and biliary disease in 178 patients undergoing ERCP. Hepatobiliary Pancreat Dis Int 2007; 6: 299-302.

18. Christoforidis E, Goulimaris I, Kanellos I, et al. The role of juxtapapillary duodenal diverticula in biliary stone disease. Gastrointest Endosc 2002; 55: 543-7.

19. Miyazaki S, Sakamoto T, Miyata M, et al. Function of the sphincter of Oddi in patients with juxtapapillary duodenal diverticula: evaluation by intraoperative biliary manometry under a duodenal pressure load. World J Surg 1995; 19: 307-12. 